\title{
DISTRIBUCIÓN ESPACIAL Y DENSIDAD DE POBLACIONES DE ARROZ ROJO (Oryza sativa L.) EN DOS SISTEMAS DE LABRANZA ${ }^{1}$
}

\author{
Steven Brenes Prendas ${ }^{2}$, Renán Agüero Alvarado ${ }^{3}$, Ana María Rodríguez Ruiz ${ }^{4}$
}

\begin{abstract}
RESUMEN
Distribución espacial y densidades de poblaciones de arroz rojo (Oryza sativa) en dos sistemas de labranza. A lo largo de cuatro ciclos de cultivo de arroz anegado, entre los años 2003 y 2004, en la Hacienda El Pelón de la Bajura, Liberia, Guanacaste, Costa Rica, se determinó la distribución espacial de las poblaciones de arroz rojo en los sistemas de Siembra Directa sobre Rastrojos (SDR) y en Labranza Mecanizada Convencional (LMC). Mediante levantamientos de malezas ubicados en puntos fijos a nivel de campo y con ayuda del programa Surfer, se logró construir mapas georeferenciales de dichas poblaciones. El sistema SDR logró disminuir el ritmo de crecimiento de las poblaciones, facilitando su manejo, por lo contrario el sistema LMC, favoreció: la distribución, el aumento y la densidad de las poblaciones de arroz rojo en el campo. Los mapas de georeferencia facilitarían la toma de decisiones en el manejo integrado del arroz rojo.
\end{abstract}

Palabras clave: Arroz rojo, patrón espacial, georeferencia, siembra directa sobre rastrojos (SDR), labranza mecanizada convencional (LMC).

\begin{abstract}
Spatial distribution and population density of red rice (Oryza sativa) under two tillage systems. During four crop cycles of flooded rice, during years 2003 to 2004, at Hacienda El Pelón de la Bajura, Liberia, Guanacaste, Costa Rica, the spatial distribution and density of red rice populations were recorded in mechanized conventional sowing (MCS) and direct seeding into stubble (DSS) by sampling fixed points in the field. By using the computer program Surfer, georeferred weed maps were developed. The treatment of sowing the crop through the previous rice stubble, without tillage, slowed the rate of red rice population growth and spreading in contrast to the treatment of conventional tillage previous to crop seading. Those maps may facilitate decission making regarding red rice management.
\end{abstract}

Key words: Red rice, spatial pattern, georeference, mechanized conventional sowing (MCS), direct seeding into stubble (DSS).

\section{INTRODUCCIÓN}

El arroz rojo (Oryza sativa) es la maleza más dañina en los campos arroceros de todo el mundo, debido a su agresividad y difícil manejo (Diarra 1985). El color rojizo de su pericarpio o del grano entero (debido a la presencia de antocianinas), varía dependiendo de la variedad. Esta característica no deseable en el mercado, hace que al intentar pulir el grano este se quiebre, reduciendo la calidad comercial del arroz (Smith 1981).

El control de esta maleza se dificulta debido a la similitud en género y especie con el arroz producido comercialmente, lo cual dificulta un control selectivo del arroz rojo. La posibilidad de utilizar variedades de arroz resistentes a diversos herbicidas, mediante transgénesis para el manejo de arroz rojo, es poco

\footnotetext{
1 Recibido: 9 de junio, 2005. Aceptado: 6 de febrero, 2006.

2 Asistente de Proyecto, LAMA-CIPROC, Universidad de Costa Rica (UCR). Correo electrónico: stevbren@ costarricense.cr

3 Coordinador del Proyecto, LAMA-CIPROC, Universidad de Costa Rica (UCR).

4 Coordinadora del Laboratorio de Malezas, LAMA-CIPROC, Universidad de Costa Rica (UCR).
} 
viable. Esto se debe al moderado control que se obtiene, además del aumento en los costos, ya que se deben realizar varios ciclos de aplicación y las cantidades de herbicidas a aplicar son altas en el caso del glufosinato de amonio de hasta 2,2 kg i.a/ha (Sankula et. al. 1997). Aunque existen herbicidas preemergentes con acción antigramineas, estos poseen un control parcial sobre las poblaciones de arroz rojo (Ortega 2005).

El arroz rojo es altamente competitivo con el cultivo, debido a que posee un mayor porcentaje de germinación, crecimiento, macollamiento (tallos productivos por planta), altura de planta y precocidad en la producción de grano que las variedades comerciales (Kwon et al. 1992). Diarra (1985) reporta que a densidades de 5; 108 y 215 plantas $/ \mathrm{m}^{2}$, reducen la producción de grano en un 22; 77 y $82 \%$ respectivamente, y con las mismas densidades reduce el peso seco en un 18; 66 y $68 \%$.

Según Huey y Baldwin (1978), citado por Eleftherohorinos (2002), las infestaciones de arroz rojo tienden a ser muy rápidas, debido a la gran cantidad de semilla que puede producir por planta (alrededor de 150 semillas/ panícula). Tomando en cuenta que cada planta tiene por promedio 10 tallos productivos, la cantidad de semillas aportada al banco en el suelo por cada planta en un ciclo sería de 1.500 semillas en promedio.

La incorporación y distribución del banco de semillas de arroz rojo se facilita mediante la labranza convencional, que consiste en la nivelación del terreno y varios pases de rastra. Al remover las semillas que se encuentran enterradas, se promueve su germinación y las futuras infestaciones de esta maleza en los siguientes ciclos; además, se distribuyen las poblaciones en el campo. Si bien el sistema LMC combate los propágulos que han emergido, si no se complementa con otro método de control, este esfuerzo será ineficiente (Agüero 1996), debido al ingreso masivo de semillas al banco, las cuales entran en una latencia inducida (Acosta y Agüero 2001).

Para lograr un control y manejo eficiente de estas poblaciones se requiere conocer su distribución en el campo, y para desarrollar un plan de manejo de la maleza en un área determinada, facilitando la toma de decisiones. La georeferencia de estas poblaciones - es decir su ubicación en un plano con coordenadas cartesianas, geodésicas o espaciales (Navarro 2001) con la ayuda de la geoestadística, permite realizar un control localizado de los principales puntos de infestación y un uso racional de los recursos con que se cuenta, para el manejo integrado de malezas (Donald 1994). Adicionalmente, se conocerán patrones de distribución de la maleza en el tiempo (Cardina et al. 1997).
El objetivo del trabajo fue determinar la influencia de la labranza mecanizada convencional (LMC) y la siembra directa sobre rastrojos (SDR) en la distribución y densidades de poblaciones de arroz rojo en un campo de producción de arroz comercial.

\section{MATERIALES Y MÉTODOS}

El experimento se realizó en el lote "Playitas", ubicado en la Hacienda El Pelón de la Bajura, en el cantón de Liberia, provincia de Guanacaste, en los ciclos de cultivo de enero a abril (ciclo 1) y de agosto a noviembre (ciclo 2) del año 2003 y los ciclos de marzo a junio (ciclo 3) y setiembre a diciembre (ciclo 4) del año 2004; dicho lote ha sido sembrado en ciclos continuos de arroz inundado en los últimos cinco años ${ }^{6}$. La duración de cada ciclo es de 120 días aproximadamente. Según la clasificación de zonas de vida de Holdrige (1979), el lugar corresponde a la formación de Bosque Tropical Seco. La precipitación anual se encuentra en el rango de los 1300 a $1700 \mathrm{~mm}$ y la temperatura promedio anual de $28^{\circ} \mathrm{C}$ (Herrera 1985).

\section{Metodología}

Se demarcaron dos parcelas con dimensiones de 70 x $70 \mathrm{~m}$, en una se preparó el terreno bajo el sistema de LMC, que consiste en dos pasadas de rastras, y el otro con SDR, en el cual se deja la paja entera de la cosecha anterior sobre el suelo, y se siembra con equipo de mínima labranza. El manejo de malezas se realizó con glifosato $(3 \mathrm{~kg} /$ ha de producto comercial formulado al $68 \mathrm{SG}$, en 200 1/ha), en presiembra en ambos sistemas. En el sistema LMC, se realizó una aplicación de butacloro, en preemergencia, a razón de 4 1/ha de producto comercial formulado a $60 \mathrm{EC}$, en 200 1/ha. Cada parcela se subdividió en una cuadrícula de 49 sub parcelas de 10 x 10 m. En el centro de cada sub parcela, en ambos tratamientos, se realizó un levantamiento de malezas con la ayuda de un marco de $1 \mathrm{~m}^{2}$, contando las plantas de arroz rojo presentes en dicha área. Los levantamientos se realizaron aproximadamente dos meses posteriores a la siembra, en los cuatro ciclos consecutivos de arroz comercial.

El análisis de los datos se hizo con el programa Surfer 32 (Versión 6,01) de Surface Mapping System, Golden Software ${ }^{7}$, el cual permite, mediante un

\footnotetext{
6 ERAS, G. 2004. Registros de la finca (entrevista). Hacienda el Pelón de la Bajura, Liberia, Guanacaste.

7 NAVARRO, J. 2004. Análisis de datos (entrevista). San José. Costa Rica. Universidad de Costa Rica.
} 
procedimiento de interpolación conocido como "kriging", crear mapas con un patrón de distribución espacial de las poblaciones presentes en este estudio, a lo largo de cuatro ciclos de monocultivo de arroz. Además, se realizaron pruebas de igualdad de medias mediante el estadístico t-student y el análisis de grupo respectivo.

\section{RESULTADOS Y DISCUSIÓN}

Al inicio de las evaluaciones en el ciclo 1, las condiciones presentes en las parcelas tanto de SDR como de LMC eran muy parecidas con respecto al número de plantas de arroz rojo y no existieron diferencias estadísticas significativas al realizar un análisis estadístico grupal de las evaluaciones realizadas en cada ciclo (Cuadro 1). Esta condición es deseable, pues se partió de una condición homogénea lo que facilitó determinar y comparar el impacto de las tecnologías bajo estudio, en la dinámica del arroz rojo.

A partir del segundo ciclo y en los siguientes, las poblaciones de arroz rojo fueron mayores en forma significativa en el sistema de LMC (Cuadro 1).

Cuadro 1. Promedio de plantas de arroz rojo (Oryza sativa) por $\mathrm{m}^{2}$ en cuatro ciclos de monocultivo de arroz, bajo los sistemas de siembra directa sobre rastrojos (SDR) y labranza mecanizada convencional (LMC). Guanacaste, Costa Rica. 2003-2004.

\begin{tabular}{lllll}
\hline Sistema de & \multicolumn{4}{c}{ Ciclos de siembra } \\
\cline { 2 - 5 } siembra & Ciclo 1 & Ciclo 2 & Ciclo 3 & Ciclo 4 \\
\hline LMC & $0,44 \mathrm{a}^{*}$ & $3,74 \mathrm{a}$ & $6,08 \mathrm{a}$ & $45,99 \mathrm{a}$ \\
SDR & $0,58 \mathrm{a}$ & $0,59 \mathrm{~b}$ & $1,7 \mathrm{~b}$ & $3,44 \mathrm{~b}$ \\
\hline
\end{tabular}

(*) Medias con igual letra en la columna son estadísticamente iguales (DMS, $\mathrm{p} \leq 0,05)$.

En el segundo ciclo de evaluaciones, el sistema SDR tendió a concentrar las poblaciones de arroz rojo (Figura 1), además mostró una tasa menor de aumento de individuos por metro cuadrado (Cuadro 1). Por el contrario, en LMC, el sistema favoreció la distribución de las poblaciones y el aumento de la densidad de los individuos, con respecto al ciclo 1 .

Cuando en LMC se voltea el suelo, se trasladan las semillas de arroz rojo de los horizontes inferiores del suelo, las cuales están listas para germinar y a su vez introduce nuevas semillas que aumentan el banco de semillas presente en el suelo. Además de promover la germinación, estas labores distribuyen el banco de semillas en el campo, con un aumento espacial de las poblaciones, como se observa a lo largo de los ciclos de LMC (Figura 1).

Para el tercer ciclo de evaluación (Figura 1), la densidad en el sistema SDR aumentó en relación con el ciclo anterior; sin embargo, la tasa de aumento fue menor en relación con la LMC (Cuadro 1). Los nuevos parches que aparecen en el mapa de SDR, se deben a la distribución de semillas de arroz rojo, proveniente de los puntos del ciclo anterior, que al no ser controlados - como se mencionó anteriormente - se dispersan por la acción del viento, la lluvia, o por la maquinaria durante la cosecha.

En el sistema LMC, los puntos que aparecen en el ciclo 3, se unen formando parches de dimensiones entre los 15 a $30 \mathrm{~m}^{2}$. Estos parches pueden alcanzar densidades de hasta 65 plantas adultas de arroz rojo por metro cuadrado, dificultando el control dirigido a estos puntos, obligando a realizar un control generalizado.

En el ciclo 4 en ambos sistemas se llega a una situación extrema (Figura 1), debido a que las poblaciones de arroz rojo en LMC son tan densas, que tienden a excluir al arroz comercial quedando solamente el arroz rojo, con densidades que alcanzan hasta las 340 plantas $/ \mathrm{m}^{2}$. En SDR los parches de arroz rojo se incrementaron en densidad y en número, alcanzando densidades inaceptables de 160 plantas $/ \mathrm{m}^{2}$, esto debido a la falta de un control complementario en los ciclos anteriores.

\section{CONCLUSIONES}

1. El sistema de siembra directa sobre rastrojos disminuye el ritmo de crecimiento de las poblaciones de arroz rojo, concentrándolas en áreas relativamente pequeñas en los primeros ciclos productivos lo cual permitirá integrar diferentes prácticas de manejo de malezas para su control, necesarias, ya que por sí sola, la SDR no controla satisfactoriamente al arroz rojo a largo plazo.

2. La labranza mecanizada convencional favorece la distribución, aumento rápido de áreas infestadas y densidad de las poblaciones de arroz rojo, lo cual agrava y encarece su manejo.

3. La georeferencia que provee los mapas de distribución espacial, es una herramienta útil para valorar las poblaciones de arroz rojo en una parcela en 

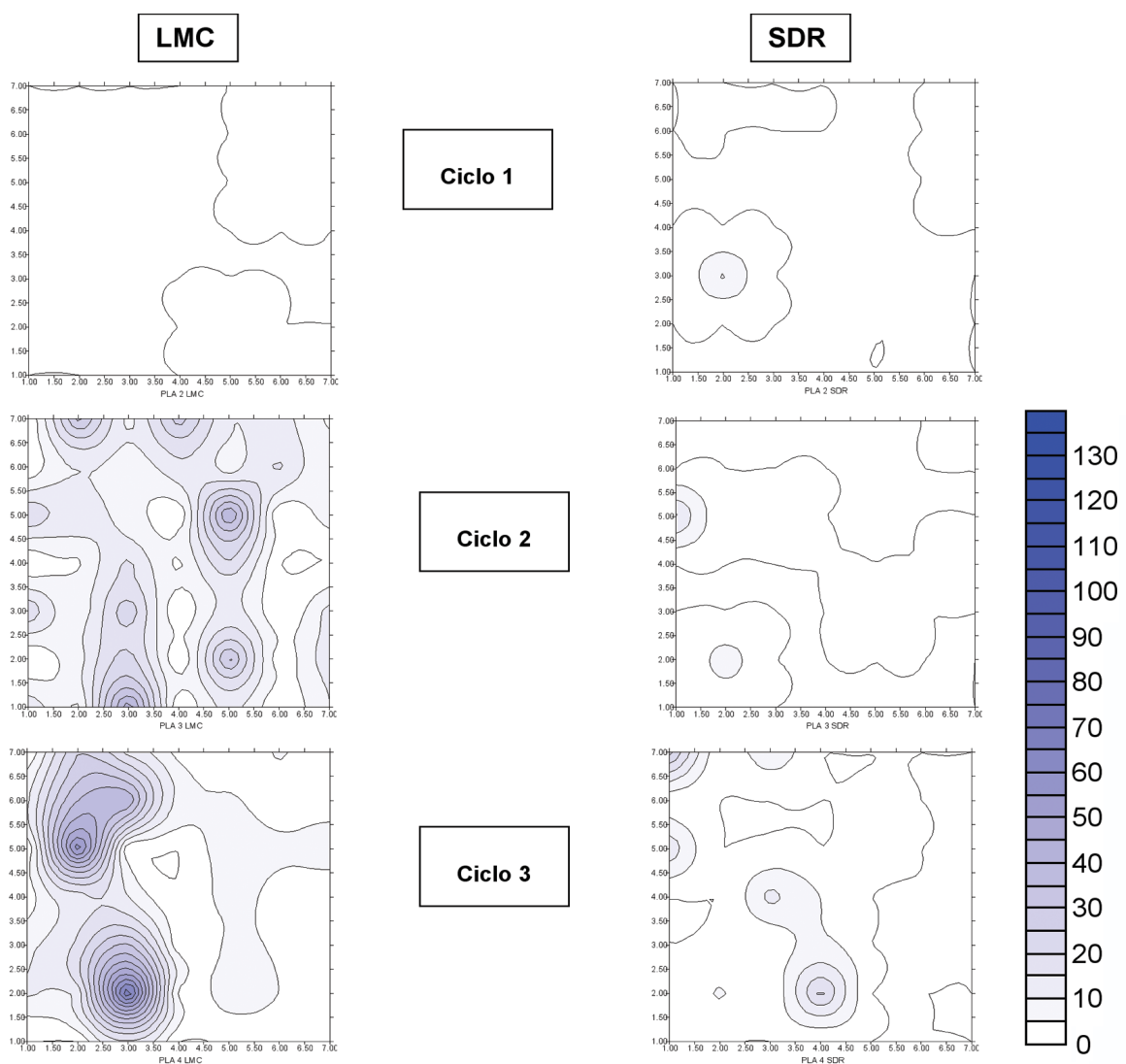

120
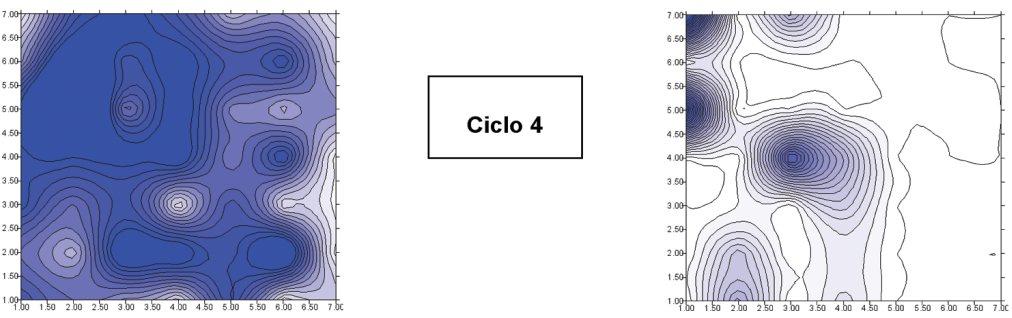

\# plantas $/ \mathrm{m}^{2}$

Ciclo 4

Figura 1. Distribución espacial de las poblaciones de arroz rojo, a lo largo de cuatro ciclos de cultivo, bajo dos sistemas de labranza. Las unidades se expresan en número de plantas de arroz rojo $/ \mathrm{m}^{2}$. Guanacaste, Costa Rica. 2003-2004.

particular. Sería recomendable adaptar esta metodología para georeferenciar a nivel de lote, para apoyar la toma de decisiones en el manejo integrado del arroz rojo.

\section{AGRADECIMIENTOS}

Agradecemos al M.Sc. Juan Ramón Navarro por la ayuda en la elaboración de los mapas geoestadísticos. A Leonel Fernández y Gonzalo Eras, funcionarios de la
Hacienda El Pelón de la Bajura sin su valiosa ayuda y generosidad no hubiese sido posible el presente estudio.

\section{LITERATURA CITADA}

ACOSTA, L.; AGÜERO, R. 2001. El banco de propágulos de malezas en el agroecosistema: conocimiento actual y propuesta metodológica para su estudio. Agronomía Mesoamericana 12(2): 141- 151. 
AGÜERO, R. 1996. Malezas del arroz y su manejo. Centro de Investigaciones en Protección de Cultivos. Universidad de Costa Rica. San José, Costa Rica. 246 p.

CARDINA, J.; JOHNSON, G.; SPARROW, D. 1997. The nature and consequence of weed spatial distribution. Weed Sci. 45: 364 - 373 .

DIARRA, A.; SMITH, R.; TALBERT, R. 1985. Interference of Red Rice (Oryza sativa) with Rice (Oryza sativa). Weed Sci. 33: 644 - 649.

DONALD, W.1994. Geostatistics for mapping weeds, with Canada thishtle (Cirsium arvense) patch as a case study. Weed Sci. 42: 648- 657.

ELEFTHEROHORINOS, I.; DHIMA, K. 2002. Red rice (Oryza sativa) with preemergence and postemegente herbicides. Weed Technol. 16: 537- 540.

HERRERA, W. 1985. Clima de Costa Rica. Vegetación y clima de Costa Rica. Ed. UNED. Vol 2. San José. Costa Rica. 118 p.

HOLDRIGE, L. 1979. Ecología basada en zonas de vida. IICA. San José. Costa Rica. 216 p.
KWON, S.; SMITH, R.; TALBERT, R. 1992. Comparative growth and development of red rice (Oryza sativa) and rice (Oryza sativa). Weed Sci. 40: 57- 62.

NAVARRO, C. 2001. Algunas aplicaciones de la geoestadística a la investigación de los patrones de distribución de agentes fitopatógenos en el cultivo de arroz en Cañas, Guanacaste. Trabajo final de Graduación para optar por Título de Licenciatura. Escuela de Fitotecnia. Facultad de Agronomía. Universidad de Costa Rica.

ORTEGA, Y. 2005. Integración de la siembra directa sobre rastrojos al manejo de arroz rojo en un agroecosistema arrocero inundado. Trabajo final de Graduación para optar por el grado de Philosophy Doctor. Sistema de Estudios de Posgrado, Doctorado en Sistemas de Producción Agrícola Tropical sostenible. Universidad de Costa Rica. 78 p.

SANKULA, S.; BRAVERMAN, M.; LINSCOMBE, S. 1997. Glufosinate - Resistant, BAR - Transformed rice (Oryza sativa) and red rice (Oryza sativa) responseto glufosinate alone and mixtures. Weed Technol. 11: 662-666.

SMITH, R. 1981. Control of red rice (Oryza sativa) in waterseeded rice (O. sativa). Weed Sci. 29: 663- 666. 